PROCEEDINGS OF THE

AMERICAN MATHEMATICAL SOCIETY

Volume 127, Number 9, Pages 2587-2590

S 0002-9939(99)04508-6

Article electronically published on April 9, 1999

\title{
SUR LES $p$-CORPS
}

\author{
M. N'KANZA
}

(Communicated by Lance W. Small)

\begin{abstract}
Here we give new examples of fields in characteristic 2 whose $u$ invariant and $\hat{u}$-invariant are different: $u(K)=2, \hat{u}(K)=2^{m}$ or $\infty(m \in$ $\mathbb{N}, m \geq 1)$. These fields are also $p$-fields.

RÉSUMÉ. Nous donnons ici de nouveaux exemples de corps $K$ en caractéristique 2 dont le $u$-invariant et le $\hat{u}$-invariant diffèrent. Plus précisément: $u(K)=2$ et $\hat{u}(K)=2^{m}$ ou $\infty(m \in \mathbb{N}, m \geq 1)$. Ces corps sont aussi des $p$-corps.
\end{abstract}

\section{INTRODUCTION}

Soit $\overline{\mathbb{Q}}$ une clôture algébrique des rationnels et $a \in \overline{\mathbb{Q}} \backslash \mathbb{Q}$. Soit encore $E$ le sous-corps de $\overline{\mathbb{Q}}$ maximal pour la propriété de ne pas contenir a (l'existence de $E$ est assurée par le lemme de Zorn). Ce corps $E$ a la propriété remarquable que toute extension finie est cyclique, c.à.d si $[M: E]<\infty$ alors $M$ est une extension galoisienne de $E$ et $\operatorname{Gal}(M / E)$ est cyclique. Moresi s'est inspiré de cette construction (voir $[\mathrm{M}]$ ), qui est due à Artin (voir [L, p.230, ex.3]), pour fournir les premiers exemples de corps $K$ de caractéristique 2 tels que $u(K)=2$ et $\hat{u}(K)=$ $2^{m}(m \in \mathbb{N}, m \geq 1)$ ou $\hat{u}(K)=\infty$. Rappelons que par définition:

$$
u(K)=\max \{\operatorname{dim} q: q \text { forme quadratique non singulière anisotrope sur } K\}
$$

et

$$
\hat{u}(K)=\max \{\operatorname{dim} q: q \text { forme quadratique anistrope sur } K\} .
$$

Dans le paragraphe 2 nous généralisons encore cette construction pour fournir de nouveaux exemples de corps de caractéristique 2 ayant la propriété précédente. D'autre part cette généralisation donne aussi des exemples de $p$-corps. Cette notion de $p$-corps a été introduite par Pfister. On dit qu'un corps $K$ est un $p$-corps, $p$ étant premier, si $[L: K]$ est une puissance de $p$ pour toute extension finie $L$ de $K$. Pfister a montré (voir $[\mathrm{P}]$ ) que ces $p$-corps ont des propriétés arithmétiques intéressantes et a énoncé la conjecture suivante: Si tout système de formes quadratiques $q_{1}, \ldots, q_{r}$ définies sur $K$ en $n$ variables, $n>r$, a une solution non triviale sur $K$ alors $K$ est un $p$-corps. Cette conjecture a été récemment résolue par $D$. Leep en caractéristique 0 (voir [Le]) et est toujours ouverte en caractéristique $>0$.

Received by the editors April 2, 1997 and, in revised form, November 18, 1997.

1991 Mathematics Subject Classification. Primary 11Exx.

Je remercie le Professeur P. Mammone pour les nombreux entretiens que nous avions eus ensemble lors de la préparation de ce travail-ci de ma thèse annexe (présentée le 26 Juin 1991 à l'Université de Mons).

(C)1999 American Mathematical Society 


\section{RÉSultats SUR les CORPS $\left(a_{1}, \ldots, a_{n}\right)$-MaXimaux}

2.1. Notions. Soit $K$ un corps de caractéristique $p$. Notons par $\wp$ le morphisme additif de $K$ dans $K$ qui envoie $x$ sur $\wp(x):=x^{p}-x$. L'ensemble $\wp(K)$ est non seulement un sous-groupe additif de $K$ mais puisque pour $b \in F_{p}$, le corps premier, et $x \in K$ on a $b .\left(x^{p}-x\right)=(b x)^{p}-b x \in \wp(K)$, le sous-groupe additif $\wp(K)$ est aussi un espace vectoriel sur $F_{p}$. On peut donc considérer le quotient $K / \wp(K)$ comme un espace vectoriel sur $F_{p}$. Si $a_{1}, \ldots, a_{n} \in K$, on notera $\left\langle\bar{a}_{1}, \ldots, \bar{a}_{n}\right\rangle_{K}$ le sous-espace vectoriel de $K / \wp(K)$ engendré par les éléments $a_{1}+\wp(K), \ldots, a_{n}+\wp(K)$.

Définition. Soit $K$ un corps de caractéristique $p$ et $a_{1}, a_{2}, \ldots, a_{n} \in K$. On dit que $K$ est $\left(a_{1}, a_{2}, \ldots, a_{n}\right)$-maximal si pour toute extension séparable finie $L$ de $K$ on a:

$$
\operatorname{dim}_{F_{p}}\left\langle\bar{a}_{1}, \ldots, \bar{a}_{n}\right\rangle_{K}=n \text { et } \operatorname{dim}_{F_{p}}\left\langle\bar{a}_{1}, \ldots, \bar{a}_{n}\right\rangle_{L}<n
$$

pour toute extension séparable finie $L$ de $K$. Bien-entendu $\left\langle\bar{a}_{1}, \ldots, \bar{a}_{n}\right\rangle_{L}$ désigne le sous-espace vectoriel de $L / \wp(L)$ engendré par $a_{1}+\wp(L), \ldots, a_{n}+\wp(L)$. Dans [M] Moresi avait considéré le cas $n=1$ et pour $a \in K \backslash \wp(K)$ avait défini le corps $K$ comme $a$-maximal si pour toute extension séparable finie $L$ de $K, \wp^{-1}(a) \in L$. La définition ci-dessus généralise donc celle de Moresi.

Le lemme suivant sera utilisé pour démontrer que si $K$ est $\left(a_{1}, \ldots, a_{n}\right)$-maximal alors $K / \wp(K)=\left\langle\bar{a}_{1}, \ldots, \bar{a}_{n}\right\rangle_{K}$.

\subsection{Résultat préliminaire.}

Lemme 1. Soit $K$ un corps de caractéristique $p$ et $a, b \in K$. Si $\operatorname{dim}_{F_{p}}\langle\bar{a}, \bar{b}\rangle_{K}=2$ alors $a \notin \wp\left(K\left(\wp^{-1}(b)\right)\right)$.

Démonstration. Posons $T:=\wp^{-1}(b)$. Un élément quelconque de $\wp(K(T))$ s'écrit $(*)$

$$
\left(\wp\left(l_{0}\right)+r(0)\right)+\left(\wp\left(l_{1}\right)+r(1)\right) T+\cdots+\left(\wp\left(l_{p-2}\right)+r(p-2)\right) T^{p-2}+\wp\left(l_{p-1}\right) T^{p-1}
$$

pour certains $l_{0}, l_{1}, \ldots, l_{p-1} \in K$ et où la fonction $r_{p}$ est définie par la formule

$$
r(i)=\sum_{j=i+1}^{p-1}\left(\begin{array}{c}
j \\
i
\end{array}\right) l_{j}^{p} b^{j-i} \text { avec } i=0,1, \ldots, p-2,
$$

et où

$$
\left(\begin{array}{c}
j \\
i
\end{array}\right)=\frac{j !}{i !(j-i) !}
$$

Supposons maintenant que $a \in \wp(K(T))$. L'élément a est donc de la forme $\left(^{*}\right)$ pour certains $l_{1}, l_{2}, \ldots, l_{p-1}$ dans $K$. En particulier ceci nous dit que tous les coefficients de $T^{i}, i=1, \ldots, p-1$, sont nuls. On a donc $\wp\left(l_{p-1}\right)=0$ et par suite $l_{p-1} \in F_{p}$. On a aussi

c-à-d $\wp\left(l_{p-2}\right)=l_{p-1} b$.

$$
\wp\left(l_{p-2}\right)+(p-1) l_{p-1}^{p} b=0,
$$

Puisque $b \notin \wp(K)$ on voit que $l_{p-1}=0$ et par conséquent $l_{p-2} \in F_{p}$. En continuant de cette manière on arrive à:

$$
l_{p-1}=l_{p-2}=\ldots=l_{2}=0 \text { et } l_{1} \in F_{p} .
$$

On a donc: $a=l_{0}^{p}-l_{0}+l_{1} b$. Ce qui contredit l'hypothèse: $\operatorname{dim}_{F_{p}}\langle\bar{a}, \bar{b}\rangle_{K}=2$. 
Théorème 2. Soit $K$ un corps de caractéristique $p$ et $a_{1}, \ldots, a_{n} \in K$. Si $K$ est $\left(a_{1}, \ldots, a_{n}\right)$-maximal alors

(a) $K / \wp(K)=\left\langle\bar{a}_{1}, \ldots, \bar{a}_{n}\right\rangle_{K}$; en particulier $K / \wp(K)$ est de dimension finie.

(b) $B r_{p}(K)=0$, la partie de p-torsion du groupe de Brauer de $K$ est triviale, c.à.d. $B r_{p}=0$.

(c) Pour $p=2, u(K)=2$.

Démonstration. Pour (a): soient $b \in K \backslash \wp(K)$ et $L=K\left(\wp^{-1}(b)\right)$. Comme $K$ est $\left(a_{1}, \ldots, a_{n}\right)$-maximal $\operatorname{dim}_{F_{p}}\left\langle\bar{a}_{1}, \ldots, \bar{a}_{n}\right\rangle_{L}<n$. En d'autres termes, il existe $i_{1}, i_{2}, \ldots, i_{n} \in F_{p}$ tels que $a:=i_{1} a_{1}+\cdots+i_{n} a_{n} \in \wp(L)$. Par le lemme 1 on déduit alors que $\operatorname{dim}_{F_{p}}\langle\bar{a}, \bar{b}\rangle_{K}=1$ ou encore $b+\wp(K) \in\left\langle\overline{a_{1}}, \ldots, \overline{a_{n}}\right\rangle_{K}$.

(b) est une conséquence immédiate d'un théorème de Saltman qui dit que si $\operatorname{dim}_{F_{p}} K / \wp(K)<\infty$ alors $B r_{p}(K)=0$ (voir [S]). On conclut alors par (a).

Pour (c): comme $\mathrm{Br}_{2}(K)=0$, on a en particulier que toute algèbre de quaternions $[a, b)_{K}$ (c-à-d l'algèbre engendrée par $i, j$ tels que $i^{2}+i=a, j^{2}=b$ et $j i=i j+j)$ est isomorphe à $M_{2}(K)$. Puisque ceci vaut pour tout $a \in K$ et tout $b \in K^{*}$ on en déduit que la forme quadratique norme $X^{2}+X Y+a Y^{2}$ représente tout élément de $K^{*}$. Car une forme quadratique non singulière de dimension 4 est du type $b\left(X^{2}+X Y+a Y^{2}\right)+c\left(Z^{2}+Z T+d T^{2}\right)$; celle-ci est clairement isotrope.

Avant de construire des corps maximaux $K$ avec $u(K)=2$ et $\hat{u}(K)=2^{m}$ ou $\infty$, voici un lemme, d'un intérêt indépendant, que l'on utilisera dans la construction.

Lemme 3. Soit $K$ un corps de caractéristique 2 tel que $\left[K: K^{2}\right]>1$. Si $u(K)=2$ alors $\hat{u}(K)=\left[K: K^{2}\right]$.

Démonstration. $\mathrm{Si}\left[K: K^{2}\right]=n$ et si $c_{1}, c_{2}, \ldots, c_{n}$ est une base de $K$ sur $K^{2}$ on voit facilement que la forme quadratique $c_{1} X_{1}^{2}+c_{2} X_{2}^{2}+\cdots+c_{n} X_{n}^{2}$ est anisotrope. D'où: $\hat{u}(K) \geq\left[K: K^{2}\right]$. En fait puisque les seules formes quadratiques non singulières et anisotropes sont de dimension deux, c-à-d du type $a\left(X^{2}+X Y+b Y^{2}\right)$, on en déduit que les seules formes quadratiques anisotropes de dimension stictement plus grande que $n=\left[K: K^{2}\right]$ ne peuvent être que du type

$$
a\left(X^{2}+X Y+b Y^{2}\right)+c_{1} X_{1}^{2}+c_{2} X_{2}^{2}+\cdots+c_{n-1} X_{n-1}^{2} .
$$

En utilisant la proposition 1.ii) page 5 de [MMW] on voit que ceci ne peut jamais se produire. En effet s'il existait une forme quadratique anisotrope du type $(* *)$ la proposition citée impliquerait que $u(K)=2\left[K: K^{2}\right]>2$. Ce qui contredirait l'hypothèse $u(K)=2$.

2.3. Construction des corps maximaux. Soient $X_{1}, \ldots, X_{m}$ des indéterminées sur $F_{2}$ et $K=F_{2}\left(X_{1}, \ldots, X_{m}\right)$. On a: $\left[K: K^{2}\right]=2^{m}$. Fixons $n \leq m$. On a bien: $\operatorname{dim}_{F_{2}}\left\langle\overline{X_{1}}, \ldots, \overline{X_{n}}\right\rangle_{K}=n$. On peut donc considérer $L$ une clôture $\left(X_{1}, \ldots, X_{n}\right)$ maximale dans la clôture séparable de $K$.

Proposition 4. $u(K)=2$ et $\hat{u}(K)=2^{m}$.

Démonstration. Puisque $L$ est $\left(X_{1}, \ldots, X_{m}\right)$-maximal, $u(L)=2$ par le théorème 2. Donc par le lemme 3 on sait aussi que $\hat{u}(L)=\left[L: L^{2}\right]$. Mais puisque $L$ est une extension séparable de $K$ on a: $\left[L: L^{2}\right]=\left[K: K^{2}\right]=2^{m}$, voir par exemple [B, lemme 1.3].

Nous terminons en faisant remarquer que si $K$ est de caractéristique $p$ et est $\left(a_{1}, \ldots, a_{n}\right)$-maximal pour certains $a_{1}, \ldots, a_{n} \in K$ alors $K$ est un $p$-corps. 
Proposition 5. Soit $K$ un corps de caractéristique $p$ et $a_{1}, \ldots, a_{n} \in K$. Si K est $\left(a_{1}, \ldots, a_{n}\right)$-maximal, alors $K$ est un p-corps.

Démonstration. Par [K, particulierement le théorème 57] il suffit de démontrer que si $M$ est une extension finie de $K$ alors $p$ divise $[M: K]$. On peut évidemment supposer que $M$ est une extension séparable de $K$. Dans ce cas par maximalité on sait qu'il existe $a \in K \backslash \wp(K)$ tel que $K\left(\wp^{-1}(K)\right) \subset M$. D'où la thèse.

\section{Questions ouvertes (en Caractéristique 2)}

1. Si $u(K)=4$, alors qu'en est-il de $\hat{u}(K)$ ? Atteint-il la borne inférieure $\left[K: K^{2}\right]$ ou la borne supérieure $2\left[K: K^{2}\right]$ ?

2. Dans ce travail la séparation entre $u(K)$ et $\hat{u}(K)$ a lieu avec $\hat{u}(K)$ atteignant sa borne inférieure. Existe-t-il des corps $K$ pour lesquels $u(K)$ et $\hat{u}(K)$ sont différents avec

a) $u(K)$ atteignant sa borne supérieure, c.à.d $u(K)<\hat{u}(K)=2\left[K: K^{2}\right]$ ?

b) $\left[K: K^{2}\right]<\hat{u}(K)<2\left[K: K^{2}\right]$

(e.g. $\left.2<n=\left[K: K^{2}\right]<\hat{u}(K)=n+2<2\left[K: K^{2}\right]\right)$ ?

\section{REFERENCES}

[B] R. Baeza: Comparing $u$-invariants of characteritic 2 Bol.Soc.Bras.Mat. 13(1992) p. $105-$ 114. MR 84f: 10027

[Bl] A. Blanchard: Les corps non commutatifs, Coll. Sup., P.U.F., 1972. MR 50:7242

[K] I. Kaplanski: Fields and Rings, Univ. Chicago Press, 1972. MR 50:2139; MR 96a:12001

[L] S. Lang: Algebra, Addison-Wesley, 1970. MR 86j:00003 (second edition)

[Le] D. Leep: Pfister conjectures on quadratic co-fields, Journal füdie reine und angewandte Mathematik 404(1990), 209 - 220. MR 91d:11036

[MMW] P. Mammone, R. Moresi, A. Wadsworth: u-invariants of fields of characteristic 2, Math. Z. 208(1991), 335 - 347. MR 92m:11033

[M] R. Moresi:On a class of fields admitting only cyclic extensions of prime power degree. Bol. Soc. Bras. Mat.Vol 15(1984), p. $101-107$. MR 87g:12003

[P] Pfister: Systems of quadratic forms, Bull. Soc. Math. France, Mémoire 59(1979), p. 115 - 123. MR 80j:10033

[S] D. Saltman: Splittings of cyclic p-algebras, Proc. Am. Math. Soc. 62(1977), p. 223-228. MR 55:8006

Université de Mons, Faculté des Sciences, Département de Mathématique, Avenue V. Maistriau, 15, B- 7000 Mons, Belgique 